CLAWAR 2018: 21st International Conference on Climbing and Walking Robots and the Support Technologies for Mobile Machines, Panama City, Panama, 10-12 September 2018

\title{
Prospects and possibilities of using thermomechanical microrobots for solving technological tasks in space
}

N. N. Bolotnik*, V. G. Chashchukhin V. G. Gradetsky, D.V. Kozlov, I.P. Smirnov and A.A. Zhukov

Institute for Problems in Mechanics of the Russian Academy of Sciences Moscow, Russia

*E-mail: bolotnik@ipmnet.ru www.ipmnet.ru

The possibilities of microrobots for inspection and technological operations on spacecraft board and in open space are analyzed. Utilization of such robots for operations on outer hulls of spacecraft and on internal units and aggregates is discussed.

Keywords: microrobot, space, spacecraft, utilization.

\section{Introduction}

Research and development of mini- and microrobots designed for operations in space are performed in industrially developed countries, mainly in Japan, USA, Germany, France, and Russia [1-5]. Among the prospective applications of microrobots in space are inspection and monitoring operations. To be able to perform such operations the robots should be equipped with appropriate sensors. The action of the microrobots can be based on various physical principles. Depending on the types of actuators, the robots can be classified into electromagnetic, piezoelectric, electroadhesive, thermomechanical, pneumatic, electromechanical, etc. robots $[2,4]$. We suggest an analysis of application areas of space microrobots that takes into account the requirements of inspection and fault detection systems for spacecraft on-board equipment $[5,6]$. The inspection crawling microrobot with a miniature optical sensor for detecting cracks on the spacecraft body (Fig.1) produced by Company SRI International [1] provides an example of a prospective robotic system for using in space. 


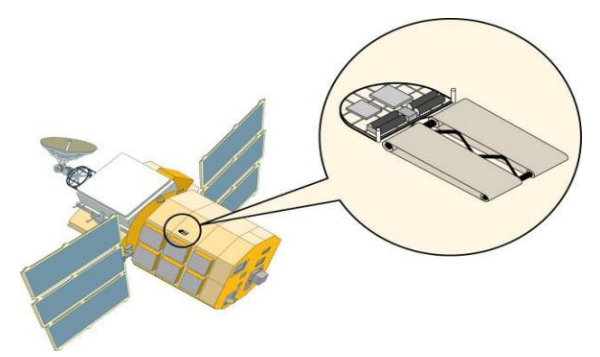

Fig. 1. Inspection crawling microrobot with a sensor for detection of cracks on the spacecraft body [1]

\section{Specific design features of inspection microrobots}

The microrobots must satisfy all requirements related to resistance of the robots to the influence of the external factors of space; the respective factors are discussed, e.g., in [6-10]. These requirements, in particular, are met by thermomechanical microrobots $[11,12]$. A justification for using such robots in space is given in the next section. Depending on the tasks to be performed, microrobots can be used separately or they can be combined into chains. The table below illustrates the correspondence between the structure of the robotic system and the tasks to be implemented.

\begin{tabular}{|l|l|}
\hline Type of the system & $\begin{array}{l}\text { Motion in narrow holes, slots, and } \\
\text { space between cables }\end{array}$ \\
\hline Separate walking microrobots & $\begin{array}{l}\text { Motion in narrow holes, slots, and } \\
\text { space between cables }\end{array}$ \\
\hline $\begin{array}{l}\text { Chains of microrobots connected } \\
\text { by flexible couplers (snake-like } \\
\text { robots) }\end{array}$ & Motion in gaps \\
\hline Chains of crawling microrobots & $\begin{array}{l}\text { Inspection of inner surfaces of } \\
\text { pipes }\end{array}$ \\
\hline Chains of walking microrobots & $\begin{array}{l}\text { Inspection of spacecraft outer sur- } \\
\text { faces }\end{array}$ \\
\hline
\end{tabular}

To be able to form chains, separate walking microrobots should have flexible coupling devices on their end faces. In addition, the microrobots should be autonomous and have an on-board power supply. 


\section{Justification of using a thermomechanical microrobot for operations in space}

We have developed a microrobotic walking platform shown in Fig. $2(a, b)$. This platform could be a prototype for developing advanced autonomous microrobotic devices for inspection of hard-to-access areas on the surface and in internal compartments of spacecraft. In addition, this platform can be used for positioning various microdevices inside spacecraft [11, 12]. A unique technology for manufacturing the microrobotic platform has been developed in Russia [5]. The walking motion of the microrobot is performed due to controlled deformation of its legs. The leg unbends when is heating and bends when is cooling. Having a mass of $70 \mathrm{mg}$, the platform can keep a load that is 20 times as large as its proper mass and move a load that 5 times exceeds the mass of the platform. The velocity of the platform with such a load is about $14 \mathrm{~mm} / \mathrm{min}$, which is rather high for mobile devices of such a type and size. For its motion the microrobot engages at least 8 legs covered with a special adhesive material, which enables the microrobot to adhere to the surface of motion in weightlessness. In a more complex design, an array of smaller legs is formed on the foot of each leg. The platform is manufactured as an integral microelectronic unit from silicon and polyimide. On one silicon plate (Fig. 2 (b)), several microrobotrs can be manufactured simultaneously by means of photolithography, sputtering, and anisotropic etching. The microrobot described is designed for operations both inside and outside of a spacecraft. The robot can operate within a temperature range of -200 to $+200 \mathrm{C}$, in the presence or absence of an atmosphere, and at low gravity. The device should also be resistant to the influence of atomic oxygen. A prototype of the walking platform of a microrobot with thermomechanical actuators [12] is shown in Fig. 3.

\section{Possible applications of microrobots for inspection of spacecraft in space}

Currently, microrobots have not found broad applications for solving various tasks on board of spacecraft, in open space or on the surfaces of other planets. This can be accounted for by both the difficulties in the creation of microrobots for solving tasks in space and the lack of justification for using such robots in space flights. Analysis of feasible applications of space microrobots suggests the following areas for them: inspection and fault detection in internal compartments of spacecraft; inspection and fault detection on outer units and surfaces of spacecraft and other structures in open space; 


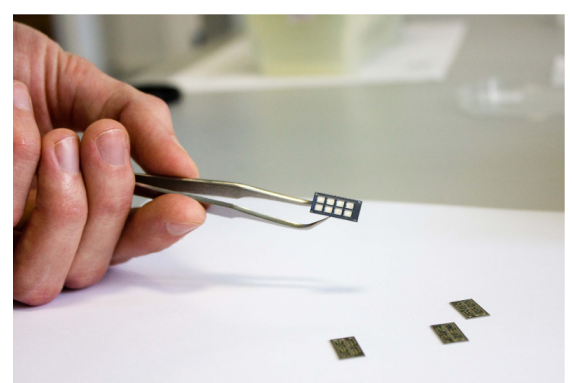

(a)

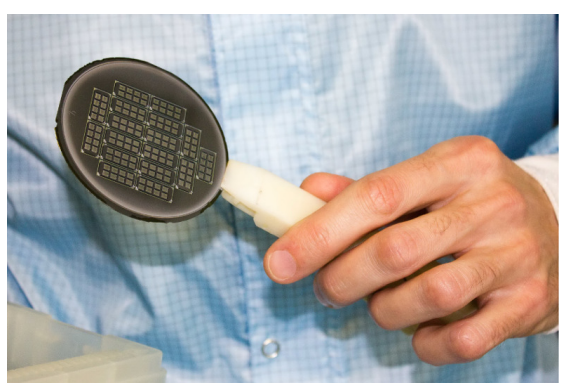

(b)

Fig. 2. (a)A microchip for a bimorph thermomechanical microrobot. (b) A silicon plate for fabricating several microrobots.

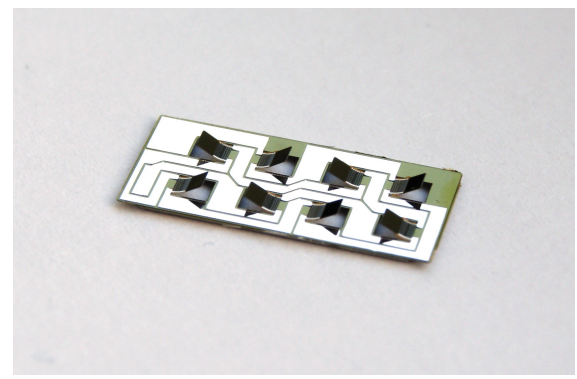

Fig. 3. A prototype of the thermomechanical walking platform [12]

operations on the surfaces of celestial bodies, including planets and large asteroids. As an example of an outer element of the structure we can consider the body of a spacecraft. The main compartments and units of a spacecraft are shown in Fig. 4. The figures indicate the areas of possible applications of microrobots. The units located in these areas are most responsible for the spacecraft and must have elevated reliability and provide a quick access for an inspection microrobot equipped with appropriate microsensors.

These areas, in particular, involve:

- Docking unit and its components (1); this unit can be inspected by a microrobot with an optical sensor.;

- Outer surface of the body of the spacecraft (2); a microrobot can be used for nondestructive inspection of the surface for detecting cracks and other damages.; 


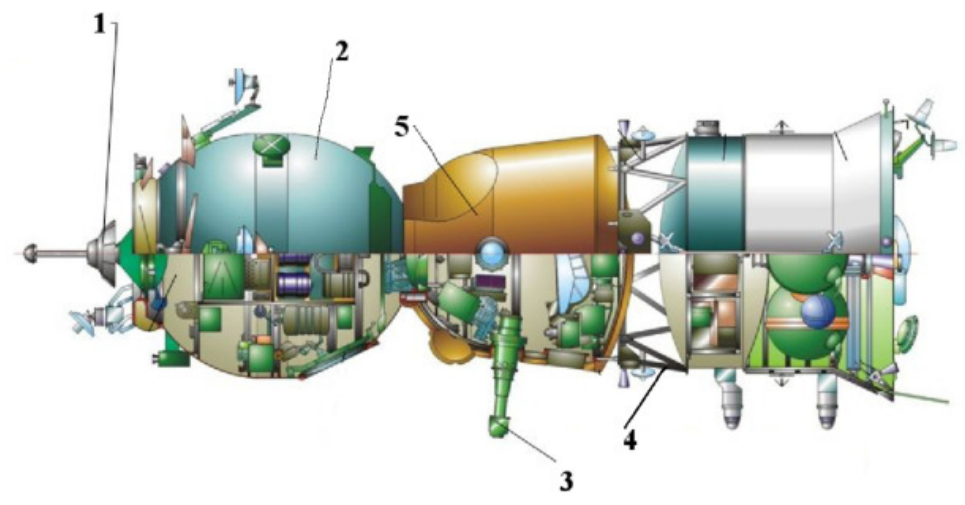

Fig. 4. Areas of possible applications of inspection microrobots on external units of a spacecraft [13]

- Optical viewfinders and other sensors of external information (3).;

- Outer components of the transfer compartment (4);

- Docking unit of the descent module (5).

\section{Utilization of microrobots in internal compartments of spacecraft}

The interior of spacecraft involves a number of structural components the inspection of which can be performed by mobile microrobots:

- ducts for laying electric cables;

- bundles of various cables;

- pipelines;

- corner compartments;

- distribution boxes;

- slits and gaps in the structure.

Operations that can be performed by microrobots involve:

- inspection of the space between cables;

- inspection of the technical state of internal compartments;

- transmission of visual information about dirt, dust, mildew, etc.;

- transmission of visual information about possible places that require a repair, about technical state of the distribution boxes, 
pipelines, cable bundles, etc.;

- cleaning;

- penetration into the slits in the inner lining;

In addition, microrobots can be used for fault detection on surfaces and coverings, as well as for inspection of solar panels.

\section{Conclusion}

- Possible application of thermomechanical microrobots for solving tasks in space is analysed.

- The solutions suggest for internal and external covers of spacecrafts.

- An information and mechanical characteristics of thermomechanical microrobots illustrate experimental possibilities of real application inside and outside of the spacecrafts.

- Thermomechanical microrobots were designed taking into account conditions of open space.

\section{Acknowledgments}

This study was supported by the Russian Science Foundation (project 1419-00949).

\section{References}

1. https://www.nasa.gov/pdf/626527main_3B-5_Pelrine.pdf.

2. W. Driesen, Concept, Modeling and Experimental Characterization of the Modulated Friction Inertial Drive (MFID). Locomotion Principle: Application to Mobile Microrobots, Computing Science Technical Report 4108, École polytechnique fédérale de Lausanne (CE 3316 (Centre Est), Station 1, CH1015 Lausanne, 2008).

3. P. Kladitis and V. Bright, Sensors and Actuators 80, 132 (2000).

4. R. D'Souza, S. Sharma, A. Pereira and A. Hashimi, AJER 5, 32 (2016).

5. I. Smirnov, D. Kozlov, A. Zhukov, V. Chashchukhin, V. Gradetsky and N. Bolotnik, Microsystem space inspector robot (versions)(October 2015), RU Patent 2566454.

6. M. Panasyuk and L. Novikova (eds.), Space Model: Physical Conditions in Space (KDU, Moscow, 2007).

7. M. Panasyuk and L. Novikova (eds.), Space Model: The Influence of the Space Environment on Materials and Equipment of Spacecraft (KDU, Moscow, 2007).

8. http://www.gctc.ru/main.php?id=940. 
9. A. Baranovsky and A. Privalov, Journal of Instrument Engineering 52, 51 (2009).

10. https://en.wikipedia.org/wiki/Outer_space.

11. N. Bolotnik, V. Gradetsky, A. Zhukov, D. Kozlov, I. Smirnov and V. Chashchukhin, Mobile microrobotics for space exploration: the state-ofthe-art and prospects, in Advances in Cooperative Robotics. Proc. of the 19th Int. Conf. of Climbing and Walking Robots, (London, UK, 2016).

12. N. Bolotnik, V. Chashchukhin, V. Gradetsky, D. Kozlov, I. Smirnov, A. Sukhanov and A. Zhukov, Design of mobile microrobots with thermomechanical actuators, in Proceed. 12th Intern. Conf. on Informatics in Control, Automation and Robotics (ICINCO-2015), (Colmar, France, 2015).

13. https://www.energia.ru/en/iss/soyuz-tma-m/soyuz-tma-m.html. 